Review began 10/12/2021 Review ended 10/19/2021 Published 10/25/2021

๑) Copyright 2021

Alajaji et al. This is an open access article distributed under the terms of the Creative Commons Attribution License CC-BY 4.0. which permits unrestricted use, distribution, and reproduction in any medium, provided the original author and source are credited.

\title{
Laboratory Abnormalities in Acne Patients Treated With Oral Isotretinoin: A Retrospective Epidemiological Study
}

Abdullah Alajaji ${ }^{1}$, Faisal A. Alrawaf ${ }^{1}$, Saleh I. Alosayli ${ }^{1}$, Hana N. Alqifari ${ }^{2}$, Bader M. Alhabdan ${ }^{1}$, Majed A. Alnasser ${ }^{1}$

1. Department of Dermatology, Qassim University College of Medicine, Buraidah, SAU 2. Department of Statistics, Qassim University College of Science, Buraidah, SAU

Corresponding author: Abdullah Alajaji, abdullahalajaji@qumed.edu.sa

\section{Abstract}

\section{Background}

Isotretinoin has been used to treat moderate to severe acne. It is well known that isotretinoin can cause an elevation in liver enzymes, triglycerides, and cholesterol. Laboratory monitoring is indicated while patients are on isotretinoin, but the frequency of laboratory monitoring is very variable among physicians who prescribe it.

\section{Study objectives}

This study aimed to determine the frequency of laboratory abnormalities of triglycerides, cholesterol, and liver aminotransferases in acne patients treated with oral isotretinoin in order to assess the need for frequent laboratory monitoring while on isotretinoin and to study the association between body weight and laboratory abnormalities.

\section{Methods}

A retrospective chart review has been conducted using data extracted from electronic medical records of the Department of Dermatology, Qassim University Medical City, Saudi Arabia. We included all acne patients who were treated with Isotretinoin for at least four months. Data were analyzed using the statistical program SPSS version 25 (Armonk, NY: IBM Corp.).

\section{Results}

A total of 407 patients met the inclusion criteria and were included in our study, 198 (48.6\%) were female and 209 (51.4\%) were males. Patients' age ranged from 10 to 51 years, with a mean age of 22.15 years. At baseline, aspartate aminotransferase (AST) was elevated in $5.4 \%$ of patients and alanine aminotransferase (ALT) was elevated in $12.7 \%$ of patients. At the last visit, AST was elevated in $3.9 \%$ of patients while ALT was elevated in $9 \%$ of patients. Triglycerides level was elevated in $12.7 \%$ of patients at the last visit compared to $6.5 \%$ of patients at baseline. Total cholesterol was elevated in $9 \%$ of patients at the last visit compared to $10.5 \%$ of patients at baseline. The increase in triglyceride levels and differences between triglycerides (TG) classifications between baseline and last visit was statistically significant $(\mathrm{P}<0.001)$. Higher body weight was associated with a higher incidence of elevation in ALT and triglycerides levels, and this association was statistically significant. There was no statistically significant relationship between total cumulative dose and laboratory abnormalities in ALT, AST, triglycerides, or total cholesterol.

\section{Conclusion}

The findings of this study indicate that oral isotretinoin can cause an elevation in ALT, AST, total cholesterol, and triglyceride levels but the incidence of these laboratory abnormalities is low and the elevation was not associated with significant morbidity, and therefore the practice of monthly laboratory monitoring for all patients while on isotretinoin needs to be revised as there is no strong evidence for such practice. We also found that patients with higher body weight are at higher risk of laboratory abnormalities and may require more frequent laboratory monitoring. Our findings support less frequent laboratory monitoring for acne patients on isotretinoin who had normal baseline labs. Frequent laboratory monitoring in these patients carries financial and emotional implications and lacks strong evidence to support this practice.

Categories: Dermatology, Epidemiology/Public Health

Keywords: cholesterol, triglycerides, liver enzymes, isotretinoin, acne vulgaris

\section{Introduction}


Acne is a very common skin condition. It is considered a follicular disease that has two stages, comedonal and inflammatory stage [1]. A comedon is formed when faulty keratinization results in a hyperkeratotic plug that blocks the opening of the pilosebaceous cell. Seborrhea (excess sebum production) causes follicular dilatation which contributes to bacterial overgrowth of Propionibacterium acne, a follicle colonizer that can cause leukocyte infiltration and follicular rupture [2]. Inflammation is triggered by follicular contents and bacterial metabolites, resulting in papules and pustules. Androgens, especially dehydroepiandrosterone sulfate (DHEAS) also contribute to acne formation. Scarring and post-inflammatory hyperpigmentation are typical residual skin changes [2]. Acne vulgaris affects 79-95\% of the teenage population [3]. Acne affects predominantly youth, but it can also affect children and adults [4].

Given aesthetic reasons, acne has a negative psychosocial effect on patients especially teenage girls, and it also can affect the quality of life [5]. Acne patients are more likely to suffer from depression, anxiety, and other psychological disorders and acne treatment can help to alleviate these symptoms [6].

Acne treatment is decided by the severity of the disease [7]. In cases of non-inflammatory acne or mild inflammatory acne, topical tretinoin, adapalene, benzoyl peroxide, azelaic acid, and topical antibiotics are commonly used, either alone or in combination. Isotretinoin is indicated for the treatment of moderate to severe inflammatory acne as well as cases of acne that are resistant to other treatment options including antibiotics and topical agents [8]. Isotretinoin doses in these cases range from 0.5 to $2 \mathrm{mg} / \mathrm{kg}$ per day, given over a 16 to 24 -week period. The usual recommended cumulative dose is $120-150 \mathrm{mg} / \mathrm{kg}$ [7]. Isotretinoin is a vitamin A-derived drug that has a powerful anti-sebum effect that reduces sebaceous gland activity and size, normalizes sebaceous follicle keratinization, and reduces the number of Propionibacterium acnes [8].

Isotretinoin has known side effects and can cause laboratory changes including elevation in liver enzymes, triglycerides (TG), and total cholesterol [7]. The most serious side effect is teratogenicity, but the most common mucocutaneous side effects are dry cracked lips, dryness of skin, and nasal mucosa.

The incidence of isotretinoin-associated laboratory abnormalities varies between different studies, and there is a wide variation in the frequency of laboratory monitoring among different prescribing physicians. The practice of monthly laboratory monitoring among isotretinoin-treated patients lacks evidence and our objective was to address this issue by studying the incidence of these laboratory abnormalities in order to see if frequent monitoring carries a large impact on clinical management on such patients or not. In addition, we studied the effect of body weight and total cumulative dose on laboratory abnormalities.

\section{Materials And Methods}

We conducted our retrospective study at the Department of Dermatology, Qassim University Medical City. Ethical approval was obtained before starting this study from Qassim University Research Ethics Committee (\#200805) on April 15, 2021.

We included all patients who received oral isotretinoin for the treatment of acne vulgaris between June 2016 and February 2021 who have completed a minimum of two isotretinoin laboratory tests during the treatment course, and these tests include aspartate aminotransferase (AST), alanine aminotransferase (ALT),

triglycerides (TG), and total cholesterol (TC). Data obtained from patient medical records included age, sex, duration of treatment, starting dose, dose at the end of the treatment, baseline labs, follow-up labs, and labs from the last visit. Statistical analysis was performed using SPSS version 25 (Armonk, NY: IBM Corp.). We reviewed laboratory results of all patients and compared labs at baseline to follow-up labs and labs at the end of the treatment course. We also studied the relationship between total cumulative dose and laboratory abnormalities. The relationship between body weight and laboratory changes during the course of treatment was also studied.

The total number of patients diagnosed with acne vulgaris was 2304 . Patients who did not receive oral isotretinoin were excluded $(n=1498)$. There were 858 patients who received oral isotretinoin but those who did not meet the inclusion criteria were excluded $(n=451)$. Thus, in the end, 407 patients were included in the study.

In this study, we evaluated changes in AST, ALT, TG, and TC levels in 407 acne patients who were treated with oral isotretinoin. Analysis of AST, ALT, TG, and TC levels was based on the National Cholesterol Education Program guidelines [9]. Aspartate aminotransferase and ALT levels were classified as normal $(<40$ $\mathrm{U} / \mathrm{L})$ and high ( $\geqslant 40 \mathrm{U} / \mathrm{L})$ [2]. Triglyceride (TG) level was classified as normal ( $\leqslant 150 \mathrm{mg} / \mathrm{dL})$ and high $(>150$ $\mathrm{mg} / \mathrm{dL})$, where total cholesterol (TC) was classified as normal $(200 \mathrm{mg} / \mathrm{dL})$ and high $(>200 \mathrm{mg} / \mathrm{dL})$.

\section{Data analysis}

Repeated measures analysis of variance was performed to compare means of laboratory values at three different intervals (baseline, follow-up, and last visit values). We used this test to study the statistical difference between the above three interval tests (baseline, follow-up, and last visit values). A paired sample t-test was used to compare means between any two groups. All tests were two-sided, with type 1 error rate 


\section{Cureus}

of 0.05 thus the results with a p-value less than 0.05 were considered statistically significant. The analysis was done with SPSS version 25 .

\section{Results}

Of the 407 patients included in our study, 198 (48.6\%) were female and 209 (51.4\%) were males (Table 1). Patients' age ranged from 10 to 51 years, with mean age of 22.15 years. The mean and (standard deviation /SD) age of the women was 22.2 (4.2) years and 22.1 (3.9) years for men (Table 1).

Demographic characteristics

Age (years)

Gende

Cumulative dose (mg)

Starting dose

Dose at end

\section{Mean (SEM)}

$22.15(0.2)$

$209(51.4 \%)$

$198(48.6 \%)$

$66.5(0.9)$

6741.94 (253.7)

$34.20(0.5)$

$37.44(0.4)$

TABLE 1: Demographic characteristics of the acne patients who had been treated with oral isotretinoin $(n=407)$.

SEM: standard error of the mean

The study evaluates the effect of isotretinoin on liver enzymes (AST and ALT) and lipids (TC and TGs).

Nearly all patients (94.6\%) had normal AST and $87.7 \%$ of them had normal ALT levels at baseline. The results are outlined in Tables 2, 3. For a few patients, there were missing individual labs in following up or last visit labs as indicated in Table 2. 


\section{Cureus}

\begin{tabular}{|c|c|c|c|c|c|}
\hline \multicolumn{6}{|l|}{ Summary of laboratory results } \\
\hline \multirow[b]{2}{*}{ Laboratory value } & \multicolumn{5}{|l|}{ Patients, n (\%) } \\
\hline & Baseline & Follow up & Last visit & score & $\begin{array}{l}\mathrm{P} \text { - } \\
\text { values }\end{array}$ \\
\hline AST: normal (<40 U/L) vs high ( $\geq 40$ U/L) & $\begin{array}{l}368(94.6 \%) \text { vs } 21 \\
(5.4 \%)\end{array}$ & $\begin{array}{l}209(94.6 \%) \text { vs } 12 \\
(5.4 \%)\end{array}$ & $\begin{array}{l}367(96.1 \%) \text { vs } 15 \\
(3.9 \%)\end{array}$ & 1.160 & 0.315 \\
\hline ALT: normal (<40 U/L) vs high ( $\geq 40 \mathrm{U} / \mathrm{L})$ & $\begin{array}{l}341(87.7 \%) \text { vs } 48 \\
(12.3 \%)\end{array}$ & $\begin{array}{l}194(88.6 \%) \text { vs } 25 \\
(11.4 \%)\end{array}$ & $\begin{array}{l}342(91.0 \%) \text { vs } 34 \\
(9.0 \%)\end{array}$ & 0.904 & 0.407 \\
\hline TC: normal ( $\leq 200$ mg/dL) vs high (>200 mg/dL) & $\begin{array}{l}357(89.5 \%) \text { vs } 42 \\
(10.5 \%)\end{array}$ & $\begin{array}{l}194(88.6 \%) \text { vs } 25 \\
(11.4)\end{array}$ & $\begin{array}{l}342(91 \%) \text { vs } 34 \\
(9 \%)\end{array}$ & 41.855 & $\leq 0.000^{*}$ \\
\hline TG: normal ( $\leq 150$ mg/dL) vs high (>150 mg/dL) & $\begin{array}{l}373(93.5 \%) \text { vs } 26 \\
(6.5 \%)\end{array}$ & $\begin{array}{l}185(83.7 \%) \text { vs } 36 \\
(16.3)\end{array}$ & $\begin{array}{l}345(87.3 \%) \text { vs } 50 \\
(12.7 \%)\end{array}$ & 20.412 & $\leq 0.000^{*}$ \\
\hline $\begin{array}{l}\text { TG: normal (<150 mg/dL) vs borderline high } \\
(150-199 \mathrm{mg} / \mathrm{dL}) \text { vs high }(200-499 \mathrm{mg} / \mathrm{dL}) \text { vs } \\
\text { very high ( } \geq 500 \mathrm{mg} / \mathrm{dL})\end{array}$ & $\begin{array}{l}373(93.5 \%) \text { vs } 18 \\
(4.5 \%) \text { vs } 8(2.0 \%) \\
\text { vs } 0(0 \%)\end{array}$ & $\begin{array}{l}184(83.3 \%) \text { vs } 23 \\
(10.4 \%) \text { vs } 13(5.9 \%) \\
\text { vs } 1(0.5 \%)\end{array}$ & $\begin{array}{l}343(86.8 \%) \text { vs } 36 \\
(9.1 \%) \text { vs } 16(4.1 \%) \\
\text { vs } 0(0 \%)\end{array}$ & & \\
\hline
\end{tabular}

\section{TABLE 2: Laboratory results of study patients ( $N=407)$.}

Some values were not recorded for all patients at each follow-up.

*Statistically significant

AST: aspartate aminotransferase; ALT: alanine aminotransferase; TC: total cholesterol; TG: triglyceride

\begin{tabular}{|c|c|c|c|}
\hline & Paired sample t-test & df & P-value \\
\hline AST: baseline-last visit & -1.726 & 364 & 0.085 \\
\hline ALT: baseline-last visit & 1.559 & 359 & 0.120 \\
\hline TC: baseline-last visit & -12.047 & 378 & $0.000^{*}$ \\
\hline TG: baseline-last visit & -6.369 & 387 & $0.000^{*}$ \\
\hline
\end{tabular}

TABLE 3: Paired samples t-test comparing baseline laboratory result to last visit result.

*Statistically significant.

AST: aspartate aminotransferase; ALT: alanine aminotransferase; TC: total cholesterol; TG: triglyceride

Aspartate aminotransferase (AST) levels were classified as normal or high. At baseline, mean (SD) AST levels were 23.8 (13.9) U/L, with normal levels in 368 (94.6\%) patients and high in 21 (5.4\%) patients. At follow-up, mean (SD) AST levels were 25.5 (9.2) U/L, with normal levels in 209 (94.6\%) patients and high levels in 12 (5.4\%) patients. At the last visit, mean (SD) AST levels were 24.9 (11.2) U/L, with normal levels in 367 (96.1\%) patients and high levels in 15 (3.9\%) patients. Aspartate aminotransferase level increased at the last visit compared to baseline and follow-up but despite this increase in values, most patients remained in the normal range during the course of treatment. Differences between AST classifications (high vs normal) at the three-time points were not statistically significant $\left(\mathrm{F}_{2}=1.160, \mathrm{df}=2, \mathrm{P}=0.315\right)$. Differences between AST levels at baseline compared to follow-up and last visit and differences between AST levels at follow-up and last visit were both not statistically significant with p-value of $0.181,0.085,0.818$, respectively. Overall, the results indicated that AST levels slightly increased over time in patients treated with isotretinoin, but the increase was not above the normal range and was not statistically significant.

Alanine aminotransferase (ALT) levels were classified as normal or high. At baseline, mean (SD) ALT level was 25.5 (15.8) U/L, with normal levels in 341 (87.7\%) patients and high in 48 (12.3\%) patients. At follow-up, 
mean (SD) ALT level was 25.4 (12.9) U/L, with normal levels in 194 (88.6\%) patients and high in 25 (11.4\%) patients. At the last visit, mean (SD) ALT level was 23.7 (16.8) U/L, with normal levels in 342 (91.0\%) patients and high in 34 (9.0\%) patients. Alanine aminotransferase levels increased at follow-up and last visit compared to baseline but despite this increase in ALT levels, it was within the normal range. Overall, ALT levels increased with time, but the differences between baseline, follow-up, and last visit were not statistically significant $\left(\mathrm{F}_{2}=0.904, \mathrm{df}=2, \mathrm{P}=0.407\right)$. The pairwise differences between ALT levels at baseline and follow-up, baseline and last visit, and follow-up and last visit were not statistically significant with $\mathrm{p}$ value of $0.567,0.120,0.245$, respectively. Overall, the results indicated that ALT levels increased over time in patients treated with isotretinoin but the increase was not statistically significant.

Total cholesterol (TC) levels were classified as normal or high. At baseline, mean (SD) TC levels were 162.4 (30.8) $\mathrm{mg} / \mathrm{dL}$, with normal levels in 357 (89.5\%) patients and high in 42 (10.5\%) patients. At follow-up, mean (SD) TC levels were 174.5 (31.6) mg/dL, with normal levels in 194 (88.6\%) patients and high in 25 (11.4\%) patients. At the last visit, mean (SD) TC levels were 176.9 (32.3) mg/dL, with normal levels in 342 (91\%) patients and high in 34 (9\%) patients. Baseline TC levels increased compared to follow-up and this was statistically significant $(\mathrm{P}<0.000)$. Differences in TC levels last visit versus baseline were statistically significant $(\mathrm{P}<0.000)$. However, changes in TC levels from follow-up compared to last visit were not statistically significant $(\mathrm{P}=0.324)$. The difference between TC classifications at each time point was statistically significant $\left(\mathrm{F}_{2}=41.855, \mathrm{df}=2, \mathrm{P}<0.000\right)$. Overall, $\mathrm{TC}$ levels at follow-up were higher than baseline, and this increase in TC levels was above the normal range.

Triglycerides (TG) levels were classified as normal or high. At baseline, mean (SD) TG level was 80.7 (30.8) $\mathrm{mg} / \mathrm{dL}$, with normal levels in 373 (93.5\%) patients and high in 26 (6.5\%) patients. At follow-up, mean (SD) TG levels were 101.6 (67.1) mg/dL, with normal levels in 185 (83.7\%) patients and high in $36(16.3 \%)$ patients. At last visit, mean (SD) TG levels were 96.1 (51.4) mg/dL, with normal levels in 345 (87.3\%) patients and high in 50 (12.7\%) patients. Triglyceride levels increased and differences between TG classifications at each time point (baseline, follow-up, and last visit) were statistically significant $\left(F_{2}=20.412, d f=2, P<0.000\right)$. TG levels increased at follow-up and last visit compared to baseline TG levels and this increase was statistically significant $(\mathrm{P}=0.000)$. However, changes in TG levels from follow-up compared to the last visit were not statistically significant $(\mathrm{P}=0.404)$. Overall, $\mathrm{TG}$ levels increased from baseline during isotretinoin treatment at follow-up and last visit, and these increases were above the normal range.

There was no significant relationship between body weight (BW) and AST levels at follow-up and last visit (Table 4). The difference in ALT levels at follow-up and last visit versus BW at baseline was statistically significant $(\mathrm{P}=0.030$ and $\mathrm{P}=0.001)$. TC levels compared to $\mathrm{BW}$ were not statistically significant for follow-up and last visit levels with p-value of $0.107,0.628$, and 0.946 , respectively. TG levels compared to BW were statistically significant for follow-up and last visit levels with $\mathrm{p}$-value $\leqslant 0.005$ and $\leqslant 0.001$ (Table 5 ). There was no statistically significant relationship found between cumulative dose (CD) and AST, ALT, TC, and TG levels (Table 6). The summary of laboratory results is shown in Figure 1.

\begin{tabular}{|c|c|c|c|c|}
\hline \multirow[b]{2}{*}{ Mean and (SD) of patients } & \multicolumn{2}{|l|}{ Mean of BW } & \multicolumn{2}{|l|}{ SD BW } \\
\hline & Normal level & High level & Normal level & High level \\
\hline BW for AST baseline levels & 66.1 & 77.3 & 16.6 & 22.0 \\
\hline BW and ASI last visit levels & 66.2 & 75.8 & 16.9 & 22.7 \\
\hline BW and ALT baseline levels & 65.5 & 76.1 & 16.6 & 18.2 \\
\hline BW and ALT last visit levels & 65.5 & 76.6 & 16.9 & 18.0 \\
\hline BW and TC baseline levels & 66.1 & 70.9 & 16.9 & 17.3 \\
\hline BW and TC last visit levels & 66.3 & 66.1 & 17.4 & 15.0 \\
\hline BW and TG baseline levels & 65.8 & 81.4 & 16.5 & 19.3 \\
\hline BW and TG last visit levels & 64.8 & 77.9 & 16.5 & 16.1 \\
\hline \multicolumn{5}{|c|}{ TABLE 4: Mean values and standard deviation of body weight (BW) of patients in relation to } \\
\hline \multicolumn{5}{|c|}{ AST: aspartate aminotransferase; ALT: alanine aminotransferase; TC: total cholesterol; TG: triglyceride } \\
\hline
\end{tabular}




\section{Cureus}

\begin{tabular}{|c|c|c|c|}
\hline & Independent samples t-test & df & P-value \\
\hline BW of AST last visit levels & -1.906 & 319 & 0.058 \\
\hline BW of ALT last visit levels & -3.378 & 314 & $0.001^{*}$ \\
\hline BW of TC last visit levels & 0.068 & 324 & 0.946 \\
\hline BW of TG last visit levels & -4.896 & 329 & $0.000^{*}$ \\
\hline
\end{tabular}

TABLE 5: Independent samples t-test to test the difference in the BW and AST, ALT, TC, and TG levels.

*Statistically significant.

AST: aspartate aminotransferase; ALT: alanine aminotransferase; TC: total cholesterol; TG: triglyceride

\begin{tabular}{|c|c|c|c|}
\hline & Independent samples t-test & df & P-value \\
\hline CD of AST last visit levels & 1.196 & 376 & 0.232 \\
\hline CD of ALT last visit levels & -0.240 & 370 & 0.810 \\
\hline CD of TC last visit levels & 0.833 & 380 & 0.405 \\
\hline CD of TG last visit levels & 0.746 & 389 & 0.456 \\
\hline \multicolumn{4}{|c|}{$\begin{array}{l}\text { TABLE 6: Independent samples t-test to test the relationship between cumulative dose (CD) and } \\
\text { AST, ALT. TC, and TG levels in the last visit results. }\end{array}$} \\
\hline *Statistically significant. & & & \\
\hline \multicolumn{4}{|c|}{ AST: aspartate aminotransferase; ALT: alanine aminotransferase; TC: total cholesterol; TG: triglyceride } \\
\hline
\end{tabular}




\section{Cureus}

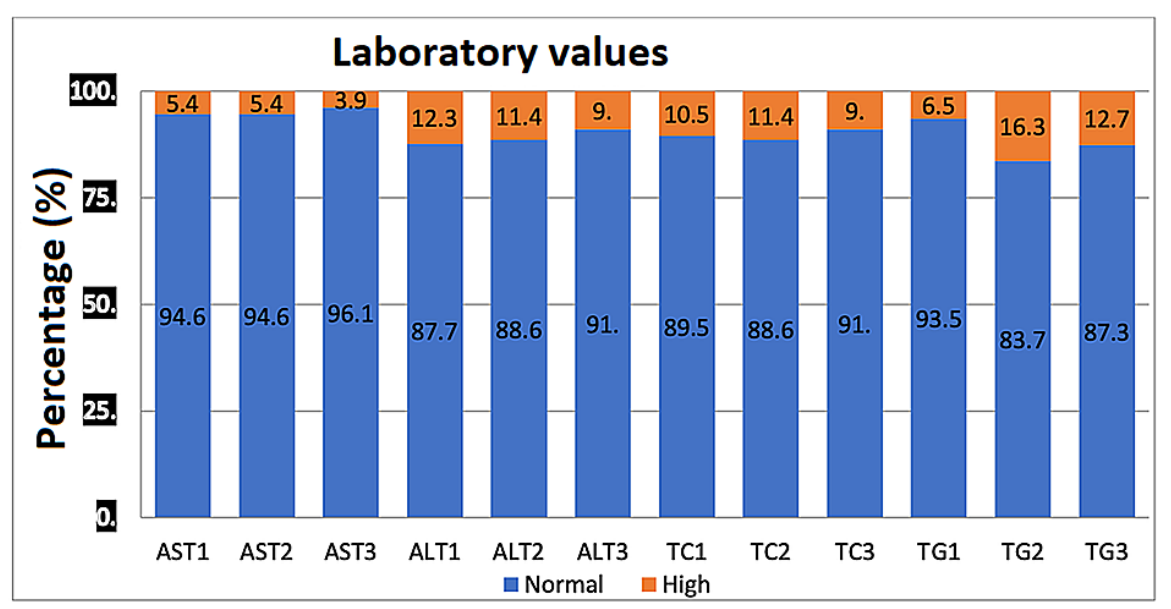

\section{FIGURE 1: Distribution of normal and abnormal laboratory values by the}

percentage of patients included in the study.

X-axis: AST1, AST2, AST3 - levels of aspartate aminotransferase at visit 1, 2, and 3 (normal vs high). ALT1, ALT2, ALT3 - levels of alanine aminotransferase at visit 1, 2, and 3 (normal vs high). TC1, TC2, TC3 - levels of total cholesterol at visit 1, 2, and 3 (normal vs high). TG1, TG2, TG3 - levels of triglycerides at visit 1, 2, and 3 (normal vs high).

Y-axis: percentage of normal vs high level of laboratory tests among patients included in the study.

AST: aspartate aminotransferase; ALT: alanine aminotransferase; TC: total cholesterol; TG: triglyceride

\section{Discussion}

We found that the incidence of abnormal elevation in ALT and AST in patients with acne treated with isotretinoin is low and was not associated with significant morbidity. Total cholesterol and triglycerides levels increased during the treatment and this was statistically significant but the increase in levels was mild. We found that patients with higher body weight are at higher risk of elevations in ALT and triglycerides levels at the end of the treatment course. The frequency of laboratory monitoring for patients on isotretinoin varies between dermatologists. Many dermatologists request monthly laboratory monitoring for their acne patients on isotretinoin.

Our study result support less frequent laboratory monitoring for healthy patients with normal baseline labs. Our findings confirmed data from other recent studies that have shown evidence that frequent monitoring of labs while on isotretinoin is not necessary for those with normal baseline labs. Shah and Kroshinsky reviewed 903 patients who were diagnosed with acne vulgaris and treated with isotretinoin therapy from 2010 to 2017, and their findings support the thought that transient alterations of lipid and liver aminotransferases levels tend to be clinically insignificant and monthly laboratory monitoring may be unnecessary [10]. A recent meta-analysis published by Lee et al. that included 1574 patients from 26 studies concluded that there is no evidence to support monthly laboratory testing for standard acne patients on standard isotretinoin dosing [11]. This meta-analysis showed that isotretinoin is associated with a statistically significant change in the mean value of several laboratory tests (white blood cell count and hepatic and lipid panels), yet high-risk or severe abnormalities are infrequent. Barbieri et al. reviewed 1863 patients treated with isotretinoin and found that grade 3 or greater triglyceride and liver function testing abnormalities were noted in fewer than $1 \%$ and $0.5 \%$ of patients screened, respectively, and authors recommended less frequent monitoring of lipid and liver function tests [12]. In a retrospective study, Hansen et al. reviewed 515 patients treated with isotretinoin and the study showed that elevation in liver transaminases was infrequent and levels were not increased significantly compared with baseline rates $(1.9 \%$ vs $1.6 \%$ at baseline) but there was significant elevations in triglyceride (19.3\%) and cholesterol (22.8\%) levels but the elevations were mild to moderate, and based on this study result, the authors recommended that in healthy patients on isotretinoin, lipid panel and liver function test be performed at baseline and second month, and further testing should be considered only if a significant abnormality in laboratory tests is noted [13]. In a recent study, Sharma and Tkachenko reviewed 735 patients and recommended less frequent laboratory monitoring for isotretinoin in healthy patients [14]. A similar conclusion was reached by Öktem et al. after reviewing 705 patients on isotretinoin [15].

Limitations of this study include the retrospective nature of this study and the fact that it is a single-center study. For few patients, there were missing individual labs from follow-up or last visit labs, and this was indicated in the tables. 


\section{Conclusions}

In conclusion, our study confirms findings from previous studies that oral isotretinoin can cause an elevation in ALT, AST, total cholesterol, and triglyceride levels. But we found that the incidence of these laboratory abnormalities is low and the elevation was not associated with significant morbidity. We also found that patients with higher body weight are at higher risk of laboratory abnormalities and may require more frequent laboratory monitoring. Our findings support less frequent laboratory monitoring for acne patients on isotretinoin who are healthy and have normal baseline labs. In these patients, especially if the dose is not increased, frequent laboratory monitoring carries financial and emotional implications and lacks strong evidence that support this practice.

\section{Additional Information \\ Disclosures}

Human subjects: Consent was obtained or waived by all participants in this study. Qassim University Research Ethics Committee issued approval \#200805. Animal subjects: All authors have confirmed that this study did not involve animal subjects or tissue. Conflicts of interest: In compliance with the ICMJE uniform disclosure form, all authors declare the following: Payment/services info: All authors have declared that no financial support was received from any organization for the submitted work. Financial relationships: All authors have declared that they have no financial relationships at present or within the previous three years with any organizations that might have an interest in the submitted work. Other relationships: All authors have declared that there are no other relationships or activities that could appear to have influenced the submitted work.

\section{References}

1. Plewig G, Kligman AM: Acne and Rosacea [Internet]. Springer Berlin Heidelberg, Berlin, Heidelberg; 2000.

2. Degitz K, Placzek M, Borelli C, Plewig G: Pathophysiology of acne. J Dtsch Dermatol Ges. 2007, 5:316-23. 10.1111/j.1610-0387.2007.06274.x

3. Rademaker M, Garioch JJ, Simpson NB: Acne in schoolchildren: no longer a concern for dermatologists . BMJ. 1989, 298:1217-9. 10.1136/bmj.298.6682.1217

4. Cordain L, Lindeberg S, Hurtado M, Hill K, Eaton SB, Brand-Miller J: Acne vulgaris: a disease of Western civilization. Arch Dermatol. 2002, 138:1584-90. 10.1001/archderm.138.12.1584

5. Shuster S, Fisher GH, Harris E, Binnell D: The effect of skin disease on self image . Br J Dermatol. 1978, 16:18-9. 10.1111/j.1365-2133.1978.tb13594.x

6. Ayer J, Burrows N: Acne: more than skin deep. Postgrad Med J. 2006, 82:500-6. 10.1136/pgmj.2006.045377

7. Vieira AS, Beijamini V, Melchiors AC: The effect of isotretinoin on triglycerides and liver aminotransferases . An Bras Dermatol. 2012, 87:382-7. 10.1590/s0365-05962012000300005

8. Kızılyel O, Metin MS, Elmas ÖF, Çayır Y, Aktaş A: Effects of oral isotretinoin on lipids and liver enzymes in acne patients. Cutis. 2014, 94:234-8.

9. Executive summary of the third report of the National Cholesterol Education Program (NCEP) expert panel on detection, evaluation, and treatment of high blood cholesterol in adults (adult treatment panel III). JAMA. 2001, 285:2486-97. 10.1001/jama.285.19.2486

10. Shah R, Kroshinsky D: Re-evaluating the need for routine laboratory monitoring in patients taking isotretinoin: a retrospective analysis. J Am Acad Dermatol. 2021, 85:504-6.

11. Lee YH, Scharnitz TP, Muscat J, Chen A, Gupta-Elera G, Kirby JS: Laboratory monitoring during isotretinoin therapy for acne: a systematic review and meta-analysis. JAMA Dermatol. 2016, 152:35-44. 10.1001/jamadermatol.2015.3091

12. Barbieri JS, Shin DB, Wang S, Margolis DJ, Takeshita J: The clinical utility of laboratory monitoring during isotretinoin therapy for acne and changes to monitoring practices over time. J Am Acad Dermatol. 2020, 82:72-9.

13. Hansen TJ, Lucking S, Miller JJ, Kirby JS, Thiboutot DM, Zaenglein AL: Standardized laboratory monitoring with use of isotretinoin in acne. J Am Acad Dermatol. 2016, 75:323-8.

14. Sharma P, Tkachenko E, Mostaghimi A: A retrospective evaluation of routine isotretinoin laboratory monitoring in patients older than 35 years. J Am Acad Dermatol. 2021, 84:201-2.

15. Öktem A, Hayran Y, Arı E, Yalçın B: Minimize the regular laboratory monitoring during the systemic isotretinoin treatment: data of 704 patients with acne vulgaris. J Dermatolog Treat. 2019, 30:813-7. 10.1080/09546634.2019.1591578 